\title{
Radiative electron capture in fast ion-atom collisions
}

\author{
D H Jakubaßa-Amundsen $\dagger$, R Höppler + and H-D Betz $\ddagger$ \\ † Physik Department, Technische Universität München, 8046 Garching, Federal Republic \\ of Germany \\ ¥Sektion Physik, Universität München, 8046 Garching, Federal Republic of Germany
}

Received 14 March 1984, in final form 29 May 1984

\begin{abstract}
Radiative electron capture (REC) has been measured in collisions between $125 \mathrm{MeV}$ sulphur ions and thin carbon-foil targets. Good agreement is found between the experimental cross section and calculations with the impulse approximation and with the strong potential Born theory.
\end{abstract}

\section{Introduction}

Radiative electron capture in ion-atom collisions was first identified by Schnopper et al (1972) and since then a large body of experimental REC data has been accumulated. Part of the interest in REC arose due to the fact that the experimental line profile $\mathrm{d}^{2} \sigma / \mathrm{d} \Omega \mathrm{d} \omega$ reflects the Doppler-broadened momentum distribution of target electrons (Spindler et al 1977). It was generally found that REC cross sections $d \sigma_{R} / d \Omega$ can be approximated reasonably well on the basis of the inverse photoeffect (Bethe and Salpeter 1957). For example, Tawara et al (1982) reproduce $\mathrm{d} \sigma_{\mathrm{R}} / \mathrm{d} \Omega$ in $\mathrm{F}^{9+} \rightarrow \mathrm{He}$ collisions within the experimental error. An improved theoretical description is expected from the impulse approximation (IA) as formulated by Kleber and Jakubaßa (1975), although total REC cross sections do not differ much from the Bethe-Salpeter results. In a recent letter Gorriz et al (1983) employed the strong potential Born (SPB) theory and arrived at the surprising conclusion that cross sections should be much larger than hitherto assumed, $\sigma^{\mathrm{SPB}} / \sigma^{\mathrm{IA}} \simeq 2 \pi Z_{\mathrm{P}} / v$, where $Z_{\mathrm{P}}$ and $v$ denote nuclear charge and velocity of the projectile ion, respectively, whereby the projectile is assumed to be the heavier collision partner. We demonstrate that this latter result can not be maintained: SPB and IA cross sections differ very little and agree well with our experimental value which has an error of less than approximately $25 \%$.

\section{Experiment}

$125 \mathrm{MeV}$ sulphur ions with initial charge states $15+$ and $16+$ were obtained from the Munich Tandem van der Graaff accelerator and passed through thin carbon targets with thicknesses between 2 and approximately $20 \mu \mathrm{g} \mathrm{cm}^{-2}$. Characteristic sulphur $\mathrm{K}$-shell $\mathrm{x}$-rays and the broad REC distribution were observed with a $\mathrm{Si}(\mathrm{Li})$ detector which could be equipped with various absorbers. Foil targets and detector were oriented at $45^{\circ}$ and $90^{\circ}$, respectively, with respect to the ion beam. Absolute radiation yields were obtained with the help of beam current measurement in a Faraday cup. 
Distortion of the REC spectrum due to electronic pulse pile-up effects was largely avoided by using absorbers and admitting only low counting rates. Target preparation and beam collimation were performed with care in order to suppress $x$-ray lines originating from bombardment of the target frame and from impurity elements contained in the self-supporting targets. Especially for the thinnest targets, however, small contributions of $\mathrm{K}$-shell $\mathrm{x}$-rays from $\mathrm{Ti}$ and $\mathrm{Fe}$ could not be entirely avoided and mask to some extent the low-intensity wings of the REC distribution.

The present collision system allows the observation of REC by ions which are either bare or have a is electron prior to capture, depending on the selection of the incident charge state. Due to the smallness of Coulomb capture cross sections from carbon (1s) into sulphur $(1 \mathrm{~s})\left(\sigma_{\mathrm{cc}} \simeq 2 \times 10^{-19} \mathrm{~cm}^{2}\right)$ and a large ratio between the lifetime of $2 \mathrm{p} \rightarrow 1 \mathrm{~s}\left(\tau=2.44 \times 10^{-14} \mathrm{~s}\right)$ and the dwell time in the target $\left(4 \times 10^{-16} \mathrm{~s}\right.$ in a $2 \mu \mathrm{g} \mathrm{cm}^{-2}$ target) an initial K-shell vacancy in the ion has a significant chance to survive passage through the target. Thorough analysis of the target-thickness dependence of both characteristic sulphur $x$-ray and REC intensities reveals that, for example, a $16+$ beam has an approximately $80 \%$ chance of not having undergone electron capture in a $2 \mu \mathrm{g} \mathrm{cm}^{-2}$ target when REC occurs and the chance to find a $1 \mathrm{~s}$ electron while the ion moves inside the target is only about $1 \%$. This situation is reflected in figure $1: 16+$ and $15+$ ions give rise to REC profiles which peak at energies characteristic for hydrogen- and helium-like final states, respectively; the peak separation of approximately $310 \mathrm{eV}$ agrees with Hartree-Fock calculations. Additional small shifts and broadening due to the aquisition of spectator electrons in about $20 \%$ of the ions are

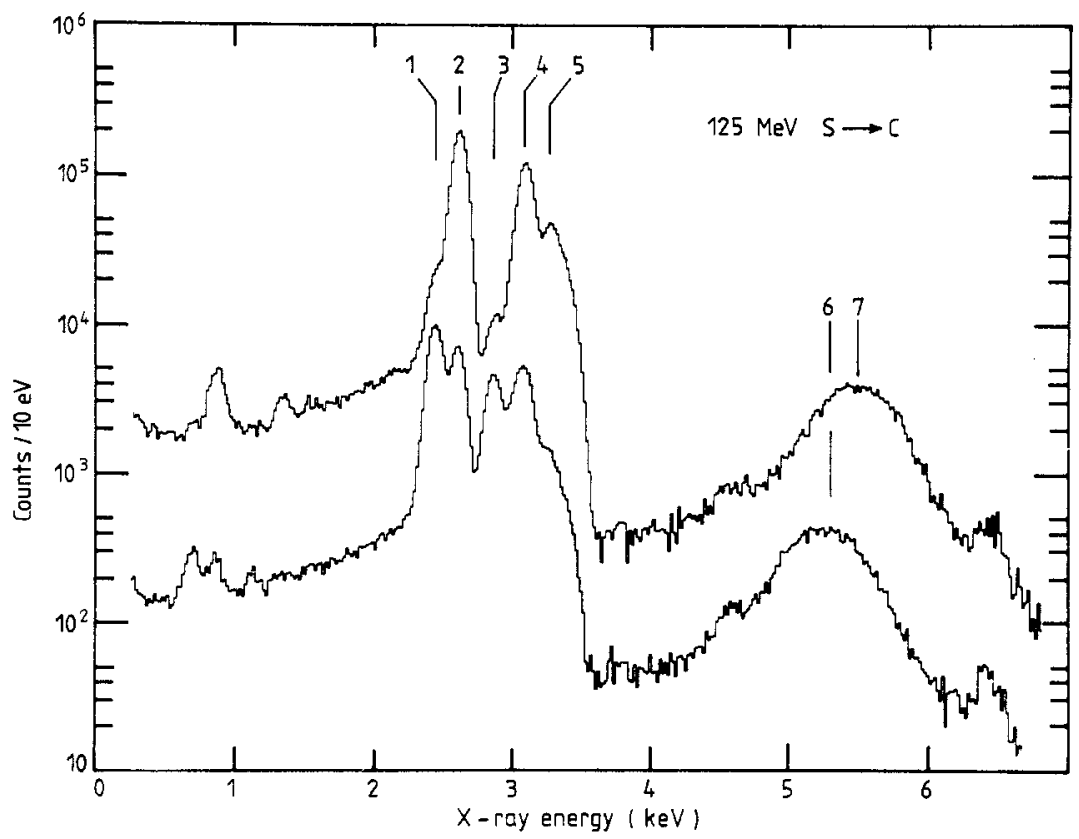

Figure 1. X-ray spectrum obtained from passage of $125 \mathrm{MeV}$ (12.5 au) sulphur ions through a $10 \mu \mathrm{g} \mathrm{cm}^{-2}$ carbon foil with incident charge $16+$ (upper curve) and $15+$ (lower curve, scaled down by a factor of ten relative to the upper curve). The detector was equipped with a $300 \mu \mathrm{m}$ carbon absorber. $1, \mathrm{~K} \alpha\left(1 \mathrm{~s} 2 \mathrm{p} \rightarrow 1 \mathrm{~s}^{2}\right) ; 2, \operatorname{Ly} \alpha(2 \mathrm{p} \rightarrow 1 \mathrm{~s}) ; 3, \mathrm{~K} \beta\left(1 \mathrm{~s} 3 \mathrm{p} \rightarrow 1 \mathrm{~s}^{2}\right)$; 4 , Ly $\beta\left(3 p \rightarrow 1 s\right.$ ) ; $5,4 p \rightarrow 1 s ; 6$, helium-like REC (final state $1 \mathrm{~s}^{2}$ ); 7 , hydrogen-like REC (final state $1 \mathrm{~s})$. 
less important and need not be discussed here. We note that a more detailed description of the experimental procedures and a comprehensive evaluation of charge and excitation states of ions inside carbon targets will be published separately (Höppler and Betz 1984).

The experimental error of the determined cross section is expected to be less than about $25 \%$. Error sources arise mainly from uncertainties in beam current measurement, solid angle of the detector, knowledge of target thickness, dead-time correction in the pulse accumulation systems, detector efficiency, absorber correction, charge-state composition of the beam, and impurities in the REC spectrum.

\section{Theory}

In a similar way as for Coulomb capture (Macek and Shakeshaft 1980) the strong potential Born theory can be applied to radiative electron capture in asymmetric collision systems. When the projectile is the heavier collision partner, the transition amplitude in the dipole approximation is given by (Gorriz et al 1983, Kleber and Jakubaßa 1975)

$$
\begin{aligned}
a_{f \mathrm{i}}=-\mathrm{i} \int_{-\infty}^{\infty} \mathrm{d} t & \int \mathrm{d} \boldsymbol{q}\left\langle\psi_{f}^{\mathrm{P}}\left|\frac{\mathrm{i}}{c} \boldsymbol{A}_{\lambda} \cdot\left(\nabla_{r}+\mathrm{i} \frac{M_{\mathrm{T}}}{M_{\mathrm{P}}+M_{\mathrm{T}}} v\right) \exp (\mathrm{i} \omega t)\right| \psi_{\boldsymbol{q}, E}^{\mathrm{P}}\right\rangle \\
& \times \varphi_{i}^{\mathrm{T}}(\boldsymbol{q}+\boldsymbol{v}) \exp (\mathrm{i} \boldsymbol{q} \cdot \boldsymbol{b}) \exp \left[\mathrm{i}\left(E_{f}^{\mathrm{P}}-E\right) t\right] \quad \boldsymbol{A}_{\lambda}=\left(\frac{c^{2}}{4 \pi^{2} \omega}\right)^{1 / 2} \boldsymbol{u}_{\lambda}
\end{aligned}
$$

where the semiclassical description has been used. A straight-line path with impact parameter $\boldsymbol{b}$ is taken for the intenuclear motion, and the radiation field is described in the projectile frame of reference. Atomic units $e=\hbar=m=1$ are used throughout. In equation (3.1) $\boldsymbol{u}_{\lambda}$ designates the polarisation direction of the emitted photon and $\omega$ its frequency, $\varphi_{i}^{\mathrm{T}}$ is the wavefunction of the bound target electron (energy $E_{i}^{\mathbf{T}}$ ) in momentum space, $\psi_{f}^{\mathrm{P}}$ stands for the final bound projectile state (energy $E_{f}^{\mathrm{P}}$ ) and $\psi_{q, E}^{\mathrm{P}}$ is a projectile off-shell continuum state with energy $E=E_{i}^{\mathrm{T}}-\boldsymbol{q} \cdot \boldsymbol{v}-v^{2} / 2$. As $\psi_{\boldsymbol{q}, E}^{\mathrm{P}}$ is non-orthogonal to $\psi_{f}^{\mathrm{P}}$, the contribution from the $\boldsymbol{r}$-independent term of the radiation field $\left(M_{\mathrm{P}}\right.$ and $M_{\mathrm{T}}$ are projectile and target mass, respectively) is finite, but as $M_{\mathrm{T}} / M_{\mathrm{P}}$ is small for asymmetric systems it may be neglected in consistency with the SPB approximation scheme.

The differential cross section for the emission of a photon into the solid angle $\mathrm{d} \Omega$ implies an integration over impact parameter and a summation over the two polarisation directions

$\frac{\mathrm{d}^{2} \sigma}{\mathrm{d} \omega \mathrm{d} \Omega}=\sum_{\lambda} \frac{(2 \pi)^{4} \omega^{2}}{c^{5} v} \int \mathrm{d} \boldsymbol{q} \delta\left(E_{f}^{\mathrm{P}}-E_{i}^{\mathrm{T}}+\omega+\boldsymbol{q} \cdot \boldsymbol{v}+\frac{1}{2} v^{2}\right)\left|\varphi_{i}^{\mathrm{T}}(\boldsymbol{q}+\boldsymbol{v})\right|^{2}\left|\boldsymbol{A}_{i}\left\langle\psi_{f}^{\mathrm{P}}\left|\nabla_{r}\right| \psi_{q, E}^{\mathrm{P}}\right\rangle\right|^{2}$.

For spherically symmetrical final states, the dipole matrix element is proportional to the momentum transfer $\boldsymbol{q}$, i.e. $\left\langle\psi_{f}^{\mathrm{P}}\left|\nabla_{r}\right| \psi_{q, E}^{\mathrm{P}}\right\rangle \equiv \boldsymbol{q} M_{E}(q)$. In this case, the summation over $\lambda$ is easily performed: when the $z$ axis is taken along $v$ such that the photon is ejected into the direction $\hat{\boldsymbol{k}}_{\lambda}=(\sin \vartheta, 0, \cos \vartheta)$ in the $x z$ plane, the two polarisation directions may be chosen as $\boldsymbol{u}_{1}=(0,1,0)$ and $\boldsymbol{u}_{2}=(-\cos \vartheta, 0, \sin \vartheta)$. Without explicit knowledge of the matrix element $M_{E}(q)$, the angular $q$ integrals can also be evaluated. The angle 
$\vartheta_{q}$ between $q$ and $v$ is determined from the $\delta$ function and for a spherical initial state the only dependence on the azimuthal angle $\varphi_{q}$ is contained in $\left(\boldsymbol{u}_{\lambda} \cdot \boldsymbol{q}\right)$. In this case,

$$
\int_{0}^{2 \pi} \mathrm{d} \varphi_{q} \sum_{\lambda}\left(\boldsymbol{u}_{\lambda} \cdot \boldsymbol{q}\right)^{2} \equiv q^{2} \pi W(\vartheta)=q^{2} \pi\left[\sin ^{2} \vartheta_{q}\left(1+\cos ^{2} \vartheta\right)+2 \cos ^{2} \vartheta_{q} \sin ^{2} \vartheta\right] \text {. }
$$

The impulse approximation is obtained from (3.2) by replacing the off-shell state by a projectile Coulomb wave $\psi_{q}^{\mathrm{P}}$ with energy $q^{2} / 2$, and for the transfer of a single target electron to a projectile $1 \mathrm{~s}$ state one obtains (without further approximations as introduced in Kleber and Jakubaßa 1975):

$$
\begin{gathered}
\frac{\mathrm{d}^{2} \sigma^{\mathrm{IA}}}{\mathrm{d} \omega \mathrm{d} \Omega}=\frac{2^{6} \omega \pi^{2} Z_{\mathrm{P}}^{5}}{c^{3} v^{2}} \int_{q_{\min }}^{\infty} q^{3} \mathrm{~d} q\left|\varphi_{i}^{\mathrm{T}}(q+\boldsymbol{v})\right|^{2} \frac{1}{\left(Z_{\mathrm{P}}^{2}+q^{2}\right)^{4}} \frac{\eta_{\mathrm{o}}\left(1+\eta_{0}^{2}\right)}{1-\exp \left(-2 \pi \eta_{0}\right)} \\
\times\left|\left[-\left(q+\mathrm{i} Z_{\mathrm{P}}\right)^{2}\right]^{-\mathrm{i} \eta_{0}}\right|^{2} W(\vartheta)
\end{gathered}
$$

with $q_{\min }=\left|E_{f}^{\mathrm{P}}-E_{i}^{\mathrm{T}}+\omega+v^{2} / 2\right| v^{-1}$ and $\eta_{0}=Z_{\mathrm{P}} / q$. The essential difference from Coulomb capture is that (3.4) has a finite limit for vanishing target field $Z_{\mathrm{T}} / r$. If the integration over photon energies $\omega$ is carried out, this limit is just the cross section for radiative recombination of a free electron (Bethe and Salpeter 1975).

As this limit is an observable quantity, any other theory for REC must have the same limiting value. In the SPB theory, the limit $Z_{\mathrm{T}} \rightarrow 0$ implies the on-shell limit of $\psi_{\boldsymbol{q}, E}^{\mathrm{P}}$, because with $\boldsymbol{q} \rightarrow-\boldsymbol{v}$ as demanded by $\varphi_{i}^{\mathrm{T}}(\boldsymbol{q}+\boldsymbol{v}), E \rightarrow v^{2} / 2 \simeq q^{2} / 2$. Then, $\psi_{\boldsymbol{q}, E}^{\mathrm{P}} \rightarrow$ $N_{0} \psi_{q}^{\mathrm{P}}$, where the normalisation constant is $N_{0}=\Gamma\left(1+\mathrm{i} Z_{\mathrm{P}} / v\right) \exp \left(\pi Z_{\mathrm{P}} / 2 v\right)$, apart from a phase factor (Okubo and Feldman 1960) which drops out after insertion into (3.2). Thus, the state $\psi_{q, E}^{P}$ has to be renormalised by $N_{0}^{-1}$ because it describes a physical scattering state (Okubo and Feldman 1960). Note that such a procedure must not be applied to Coulomb capture where the intermediate off-shell state is unobservable and where one obtains a vanishing capture cross section for $Z_{\mathrm{T}} \rightarrow 0$ (Macek and Alston 1982).

Using the methods of Macek and Alston (1982) for the evaluation of matrix elements with an off-shell function, and exploiting the relation $\psi_{\boldsymbol{q}, E}^{*(-)}=\psi_{-\boldsymbol{q}, E}^{(+)}$which connects incoming and outgoing waves, it is straightforward to calculate the exact SPB for radiative capture. Decomposing $\psi_{q, E}^{\mathrm{P}}=\chi_{\boldsymbol{q}, E}+|\boldsymbol{q}\rangle$ where $|\boldsymbol{q}\rangle$ is a plane wave, the dipole matrix element can be found from

$$
\begin{aligned}
& \left\langle\exp \left(-Z_{\mathrm{P}} r\right)\right| r^{-1} \exp (\text { is } \cdot r)\left|\chi_{q, E}\right\rangle \\
& =\frac{\mathrm{i} 2^{3 / 2}}{N_{0} \sqrt{\pi}} \frac{Z_{\mathrm{P}}^{2}}{\eta F D_{1}\left(B^{2}-C\right)^{1 / 2}}\left(\frac{1}{(1-\mathrm{i} \eta) \rho_{+}}{ }_{2} F_{1}\left(1,1-\mathrm{i} \eta, 2-\mathrm{i} \eta, \rho_{+}^{-1}\right)\right. \\
& \left.-\frac{1}{\mathrm{i} \eta}{ }_{2} F_{1}\left(1, \mathrm{i} \eta, 1+\mathrm{i} \eta, \rho_{-}\right)-\frac{\pi \mathrm{i}}{\sinh \pi \eta}\left(-\rho_{-}\right)^{-\mathrm{i} \eta}\right) \\
& \eta=Z_{\mathrm{P}} / K \quad K=(2 E+\mathrm{i} \varepsilon)^{1 / 2} \quad(\varepsilon \rightarrow 0) \\
& F=\left(Z_{\mathrm{p}}+\mathrm{i} K\right)^{2}+s^{2} \quad B=\left(F D_{1}\right)^{-1}\left(E_{1} E_{2}+4 K^{2} q \cdot s\right) \\
& D_{1}=-K^{2}+q^{2} \quad D_{2}=\left(Z_{\mathrm{P}}-\mathrm{i} K\right)^{2}+s^{2} \\
& E_{1}=-K^{2}-q^{2} \quad E_{2}=-K^{2}-Z_{\mathrm{P}}^{2}-s^{2} \\
& C=D_{2} / F \quad \rho_{ \pm}=B \pm\left(B^{2}-C\right)^{1 / 2}
\end{aligned}
$$

for a final $1 \mathrm{~s}$ state by differentiating with respect to $s$ and setting $s=0$ afterwards, and by using $\nabla_{r} \exp \left(-Z_{\mathrm{p}} r\right)=Z_{\mathrm{P}}(r / r) \exp \left(-Z_{\mathrm{p}} r\right)$. This leads to a result which has the same 
structure as for Coulomb capture (Jakubaßa-Amundsen 1984) such that the same numerical techniques can be applied for the evaluation of the hypergeometric functions ${ }_{2} F_{1}$. For a final $1 \mathrm{~s}$ state and spherical initial states one obtains

$$
\begin{aligned}
& \frac{\mathrm{d}^{2} \sigma^{\mathrm{SPB}}}{\mathrm{d} \omega \mathrm{d} \Omega}=\frac{2^{5} \pi \omega Z_{\mathrm{P}}^{5}}{c^{3} v^{2}\left|N_{0}\right|^{2}} \int_{q_{\min }}^{\infty} q^{3} \mathrm{~d} q\left|\varphi_{i}^{\mathrm{T}}(q+v)\right|^{2} W(\vartheta) \mid \frac{\mathrm{i}}{\left(Z_{\mathrm{P}}^{2}+q^{2}\right)^{2}} \\
& +\frac{Z_{\mathrm{P}}^{2}}{\eta} \frac{1}{F D_{1}\left(B^{2}-C\right)^{1 / 2}}\left[-{ }_{2} F_{1}\left(1,1-\mathrm{i} \eta, 2-\mathrm{i} \eta, \rho_{+}^{-1}\right)\right. \\
& \times \frac{1}{(1-\mathrm{i} \eta) \rho_{+}}\left(\frac{B B^{\prime}}{B^{2}-C}+\mathrm{i} \eta \frac{\rho_{+}^{\prime}}{\rho_{+}}\right) \\
& +\left({ }_{2} F_{1}\left(1, \mathrm{i} \eta, 1+\mathrm{i} \eta, \rho_{-}\right) \frac{1}{\mathrm{i} \eta}+\frac{\pi \mathrm{i}}{\sinh \pi \eta}\left(-\rho_{-}\right)^{-\mathrm{i} \eta}\right) \\
& \left.\times\left(\frac{B B^{\prime}}{B^{2}-C}+\mathrm{i} \eta \frac{\rho_{-}^{\prime}}{\rho_{-}}\right)-\frac{1}{\rho_{+}\left(\rho_{+}-1\right)} \rho_{+}^{\prime}-\frac{1}{\rho_{-}\left(1-\rho_{-}\right)} \rho_{-}^{\prime}\right]\left.\right|^{2}
\end{aligned}
$$

with $B^{\prime}=4 K^{2} / F D_{1}, \rho_{ \pm}^{\prime}=\left[1 \pm B\left(B^{2}-C\right)^{-1 / 2}\right] B^{\prime}$ and $q \cdot v=-\left(E_{f}^{\mathrm{P}}-E_{i}^{\mathrm{T}}+\omega+\frac{1}{2} v^{2}\right)$.

The poles $\omega_{n}$ of $(3.6)$ at $\mathrm{i} \eta=n(n=2,3, \ldots)$ are independent of the integration variable, $\omega_{n}=Z_{\mathrm{p}}^{2} / 2\left(1-n^{-2}\right)$ and describe the transitions of a projectile electron into the $1 \mathrm{~s}$ ground state after capture to an excited projectile state which is bound and not a continuum intermediate state (Gorriz et al 1983). If $\psi_{f}^{\mathrm{P}}$ was an excited state of main quantum number $n_{0}, n$ would be restricted to $n>n_{0}$ for $\omega_{n}>0$. It follows from (3.6) that the differential cross section diverges approximately as $\left(\omega-\omega_{n}\right)^{-2}$ near the poles, which leaves a first-order singularity in the total cross section. This rather unphysical behaviour indicates the breakdown of the SPB theory at $\omega<\omega_{\infty}=Z_{\mathrm{P}}^{2} / 2$. Then additional interactions with the (weak) target field will become important. Note, that within the validity range of SPB $\left(v \gg Z_{\mathrm{T}}\right)$, the poles lie always on the low-energy wing of the REC peak which is at $\omega_{\mathrm{P}}=\frac{1}{2} v^{2}-E_{f}^{\mathrm{P}}+E_{i}^{\mathrm{T}}$. Furthermore, the pole contributions vanish for $Z_{\mathrm{T}}=0$.

In the work of Gorriz et al (1983) a peaking approximation is applied in order to evaluate the SPB capture cross section, which is based on the fact that the target momentum function $\varphi_{i}^{\mathrm{T}}(\boldsymbol{q}+\boldsymbol{v})$ is strongly peaked at $\boldsymbol{q}=-\boldsymbol{v}$. In this approximation the off-shell intermediate state reduces to a renormalised Coulomb wave, $\psi_{q, E}^{\mathrm{P}} \simeq N_{0} \psi_{q}^{\mathrm{P}}$, so that the cross section becomes proportional to the one which results from the impulse approximation, whereby the proportionality constant is given by $\left|N_{0}\right|^{2}=$ $2 \pi Z_{P} / v\left[1-\exp \left(-2 \pi Z_{\mathrm{P}} / v\right)\right]^{-1}$. Contrary to the procedure applied in the calculation by Gorriz et al (1983) we insert the renormalised off-shell state, $N_{0}^{-1} \psi_{q, E}^{\mathrm{P}}$, into the dipole matrix element; then the peaking form of the SPB approximation becomes indeed identical to the IA as formulated in equation (3.4). The accuracy of the peaking approximation itself may be estimated from the comparison of SPB and IA (see figure 2 ) and is found to improve when the ratio $Z_{\mathrm{T}} /(n v)$ decreases, where $n$ stands for the main quantum number of the target electron to be captured. Incidentally, Gorriz et al (1983) employ the further approximation $q=(2 E)^{1 / 2}$ in the expression for $\psi_{q}^{\mathrm{P}}$ so that their result differs from the renormalised IA by a small amount which is of the same order of magnitude as the inaccuracy of the peaking approximation itself. 


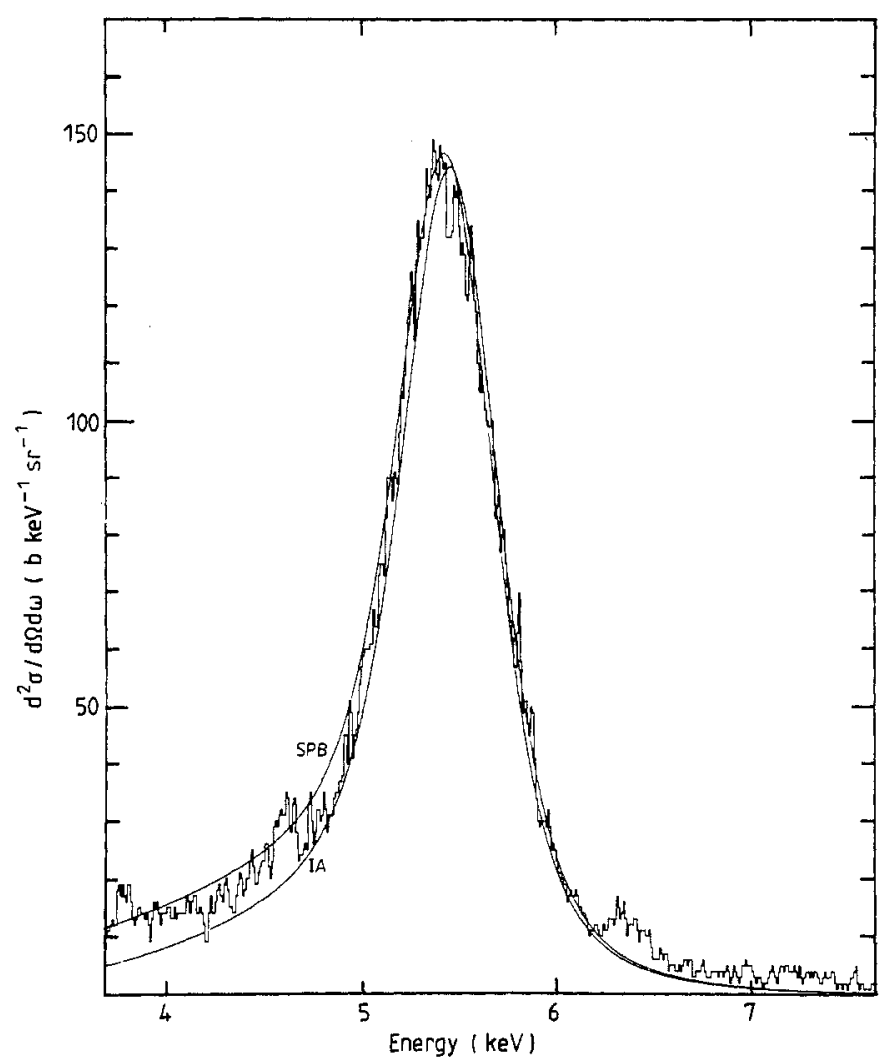

Figure 2. Comparison between experimental (histogram) and theoretical REC cross sections for the system $\mathrm{S}^{16+} \rightarrow \mathrm{C}, v=12.5$ au. IA, impulse approximation; SPB, strong potential Born approximation. The experimental spectrum has been corrected for absorber effects.

\section{Discussion}

Figure 2 shows a comparison between calculated and experimental photon spectra for $125 \mathrm{MeV} \mathrm{S}^{16+} \rightarrow \mathrm{C}$ collisions. In both calculations target states were represented by using experimental binding energies and hydrogen-like wavefunctions with effective charges of 5.62 and 1.78 for core and valence electrons, respectively. The latter values were obtained from a fit to Hartree-Fock wavefunctions and experimental Compton profiles, assuming spherically symmetric electron distributions. The general shape of the REC spectrum is reasonably well represented by both theoretical models, especially at the high-energy side. There is, however, a small difference between IA and SPB results with respect to the position and width of the peak, and a significant deviation at the low-energy side where SPB cross sections lie by a factor of up to 2.5 above the IA values. The present data are not of sufficient accuracy to distinguish safely which model is the better one. We note, though, that if the SPB had not been renormalised it would lie a factor of 8.05 higher, with the same shape as our SPB curve. In particular, the peaking version as suggested by Gorriz et al (1983) would lead to the IA curve multiplied by this factor, and produce severe disagreement with the experimental data.

In table 1 we compare our various calculations of $d \sigma_{R} / d \Omega$ with our experimental results. Agreenient is obtained within the experimental error, but it should be noted 
Table 1. REC cross sections $d \sigma_{R} /\left.d \Omega\right|_{90^{\circ}}$ for 12.5 au $S^{16+} \rightarrow C$ as obtained from various models as described in the text, compared with the experimental value.

\begin{tabular}{lccc}
\hline & $\begin{array}{l}\text { Valence } \\
\text { electrons }\end{array}$ & Core & Total \\
\hline BS $_{\mathrm{R}} / \mathrm{d} \Omega\left(\mathrm{b} \mathrm{sr}^{-1}\right)$ & 93 & 46 & 139 \\
$\mathrm{IA}$ & 90 & 36 & 126 \\
$\mathrm{SPB}^{\mathrm{b}}$ & 725 & 290 & 1014 \\
$\mathrm{SPB}$ & 94 & 48 & 142 \\
Experiment & - & - & $120 \pm 30$ \\
\hline
\end{tabular}

${ }^{a}$ Bethe and Salpeter (1957).

${ }^{b}$ Gorriz et al (1983).

that possible contributions to the spectrum such as secondary electron bremsstrahlung, unresolved $x$-ray lines from target impurities and electronic pulse pile-up have not been subtracted. We estimate that these effects could reduce the experimental cross section by about $10 \%$.

We conclude that the main REC features, peak position, total cross section and line profile in the vicinity of the peak, are well understood. The remaining questions concern the precise width of the line and the profile on the low-energy side where theoretical descriptions differ, and in the high-energy wing far from the peak $(|q+v| \gg v)$ where the measured intensities remain unexplained (Betz et al 1975) and may require the inclusion of adiabatic collision concepts. Finally, the angular distribution awaits verification as well as the forward-backward symmetry (Spindler et al 1979) at relativistic velocities.

\section{Acknowledgments}

We would like to thank $M$ Kleber for valuable commments. This work has been partially supported by the Bundesministerium für Forschung und Technologie and by GSI Darmstadt.

\section{References}

Bethe H A and Salpeter E E 1957 Encyclopedia of Physics vol 35, ed S Flügge (Berlin: Springer) p 408 Betz H D, Bell F, Panke H, Stehling W, Spindler E and Kleber M 1975 Phys. Rev. Lett. 34 1256-9

Gorriz M, Briggs J S and Alston S 1983 J. Phys. B: At. Mol. Phys. 16 L665-70

Höppler R and Betz H-D 1984 in preparation

Jakubaßa-Amundsen D H 1984 Z. Phys. A 316 161-7

Kleber M and Jakubaßa D H 1975 Nucl. Phys. A 252 152-62

Macek J and Alston S 1982 Phys. Rev. A 26 250-70

Macek J H and Shakeshaft R 1980 Phys. Rev. A 22 1441-6

Okubo S and Feldman D 1960 Phys. Rev. 117 292-306

Schnopper K W, Betz H-D, Delvaille J P, Kalata K, Sohval A R, Jones K W and Wegner H E 1972 Phys. Rev. Lett. $29898-901$

Spindler E, Betz H-D and Bell F 1977 J. Phys. B: At. Mol. Phys. 10 L561-4

- 1979 Phys. Rev. Lett. 42 832-5

Tawara H, Richard P and Kawatsura K 1982 Phys. Rev. A 26 154-61 